\title{
Size Fluctuations of Near Critical Nuclei and Gibbs Free Energy for Nucleation of BDA on $\mathrm{Cu}(001)$
}

\author{
Daniel Schwarz, Raoul van Gastel, Harold J. W. Zandvliet, and Bene Poelsema \\ Physics of Interfaces and Nanomaterials, MESA ${ }^{+}$Institute for Nanotechnology, University of Twente, \\ P.O. Box 217, NL-7500AE Enschede, Netherlands
}

(Received 20 March 2012; revised manuscript received 29 May 2012; published 6 July 2012)

\begin{abstract}
We present a low-energy electron microscopy study of nucleation and growth of BDA on $\mathrm{Cu}(001)$ at low supersaturation. At sufficiently high coverage, a dilute BDA phase coexists with $c(8 \times 8)$ crystallites. The real-time microscopic information allows a direct visualization of near-critical nuclei, determination of the supersaturation and the line tension of the crystallites, and, thus, derivation of the Gibbs free energy for nucleation. The resulting critical nucleus size nicely agrees with the measured value. Nuclei up to 4-6 times larger still decay with finite probability, urging reconsideration of the classic perception of a critical nucleus.
\end{abstract}

Different models exist that describe the nucleation process of solid structures from a dilute mother phase. All of them have the existence of a critical nucleus size in common. This critical size gives the smallest stable structure during nucleation [1-5]. Smaller nuclei tend to decay; larger nuclei tend to grow. The reason for this is a competition between positive interface energy terms, favoring decay, and negative bulk terms, favoring growth in the case of supersaturation. For epitaxial growth of metals or semiconductors at room temperature, the size of the critical nucleus is typically a handful of atoms or molecules, while at higher temperatures it can contain several hundred entities [6]. The small size at low temperatures and the fast fluctuations and high mobility at high temperatures make it challenging to observe critical nuclei experimentally. Examples of the direct observation are, therefore, rare. Subcritical nuclei were observed during the nucleation of strained $\mathrm{Ge}$ islands on a flat $\mathrm{Si}$ surface $[7,8]$. Jesson et al. observed decay of nuclei showing an unexpectedly large size (270 atoms) with STM. Another example is the nucleation of crystallites from a glassy phase, using fluctuation TEM and laser pump-probe techniques [9]. On much larger length scales, it is possible to observe the critical nucleus during the growth of colloidal crystals using laser scanning confocal microscopy [10].

The models that describe nucleation are all valid under different conditions $[1,4,11]$. For nucleation close to thermal equilibrium, i.e., low supersaturation and temperatures well below the critical temperature, a classical thermodynamic approach is appropriate. The Gibbs free energy of a randomly formed 2D cluster can easily be determined. Molecules at the islands' perimeter add a positive energy term through the edge line tension, and molecules in the bulk add a negative term due to free energy gained from lateral bonding. This leads to an energy maximum for a size where both contributions are equal which defines the critical nucleus size. For a circular flat cluster of size $A$, the Gibbs free energy is given by

$$
\Delta G=-\frac{A}{\Omega} \Delta \mu+2 \sqrt{\pi A} \chi,
$$

where $\chi$ is the cluster's edge line tension, $\Omega$ the area occupied by one molecule in the cluster, and $\Delta \mu$ the difference in chemical potential of a molecule in the dilute and in the condensed phase. In the remainder, we will refer to this quantity as chemical potential difference. The maximum, i.e., the Gibbs free energy for nucleation, is reached for a size of $A^{*}=\pi\left(\frac{\Omega \chi}{\Delta \mu}\right)^{2}$. Clusters that are smaller than this size have the tendency to decay; clusters that are larger have the tendency to grow. However, even clusters larger than the critical nucleus can still decay, due to the statistical nature of the process. In equilibrium, the density of subcritical clusters of size $A$ at a given supersaturation is given by

$$
n(A)=n_{s} \exp [-\Delta G(A) / k T],
$$

where $n_{s}$ is the density of nucleation sites. However, during growth, the system is by definition not in equilibrium, and these numbers can differ by one or two orders of magnitude [1].

Here we report on the observation of critical nuclei during the crystallization of 4, 4'-biphenyldicarboxylic acid (BDA) molecules into two-dimensional (2D) crystals on $\mathrm{Cu}(001)$ using low-energy electron microscopy (LEEM). BDA forms well ordered nanostructures on $\mathrm{Cu}(001)$ exhibiting a square $c(8 \times 8)$ superstructure [12,13]. Neighboring molecules are rotated by $90^{\circ}$ and interact laterally through hydrogen bonds. These bonds form between the carboxylate group of one molecule and the phenyl rings of the next molecule (see inset in Fig. 1). The strength of this bond is $E_{\mathrm{NN}}=0.18 \mathrm{eV}$ [14]. Surrounding the BDA islands is a molecular dilute phase. Close to room temperature, the equilibrium density in the dilute phase is around a few percent of that in the $c(8 \times 8)$ phase [14]. We will express the density $\theta$ in the remainder of this Letter in coverage of the underlying $\mathrm{Cu}$ lattice. Each molecule occupies $16 \mathrm{Cu}$-lattice sites or an area of $\Omega=1.043 \mathrm{~nm}^{2}$ and the fully completed $c(8 \times 8)$ structure corresponds then to a coverage of $0.0625 \mathrm{ML}$. 


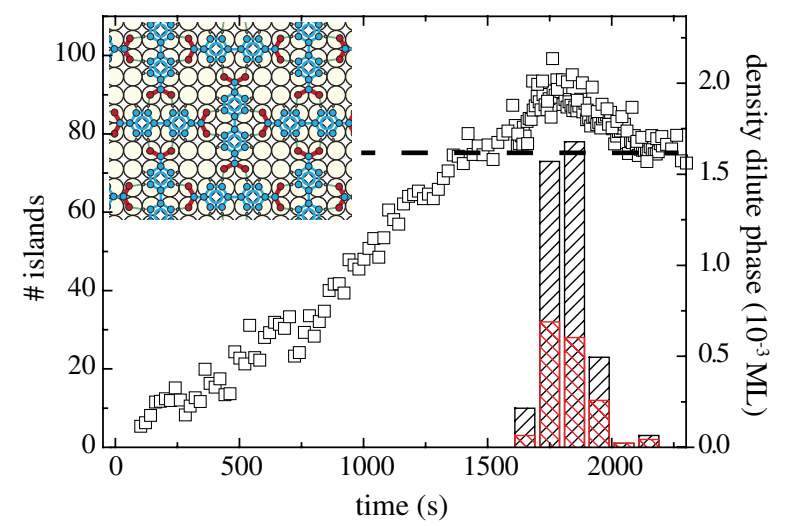

FIG. 1 (color online). Number of nucleating and decaying islands in an area of $12.5 \mu \mathrm{m}^{2}$ (bars, left axis) and density in the dilute phase (squares, right axis). The substrate temperature is $332 \mathrm{~K}$, the shutter is opened at $0 \mathrm{~s}$. From the linear increase of the gas density before nucleation, we find a deposition rate of $F=1.06 \times 10^{-6} \mathrm{ML} / \mathrm{s}$. Hatched bars show all nucleation events, cross hatched bars nuclei that decayed. The dashed line marks the equilibrium BDA density level. The inset shows the arrangement of molecules in the $c(8 \times 8)$ structure. Blue (light gray) circles stand for carbon, red (dark gray) for oxygen, small gray circles for hydrogen atoms.

We used a low BDA deposition rate of only a fraction of a ML per hour. Above room temperature, the BDA domains grew therefore at low supersaturation, i.e., close to thermal equilibrium. Under these conditions, classical thermodynamic nucleation theory should be applicable [1].

Experiments were performed in an Elmitec LEEM III with a base pressure in the low $4 \times 10^{-10}$ mbar range. A $\mathrm{Cu}(001)$ single crystal was annealed outside UHV for $48 \mathrm{~h}$ at $1173 \mathrm{~K}$ under a flow of a $\mathrm{Ar} / \mathrm{H}_{2}$ gas to deplete bulk sulfur and carbon contamination. The surface was further prepared by prolonged cycles of sputtering with hydrogen and argon ions and subsequent annealing at $873 \mathrm{~K}$. Images were recorded in bright-field mode with an electron energy of $2 \mathrm{eV}$ with a frame rate of $0.5 \mathrm{~Hz}$. These settings did not lead to any observable electron damage to the molecules. All images were corrected for a background originating from the MCP detector plates.

We measured the density in the dilute phase by analyzing locally the change of image intensity on the $\mathrm{Cu}$ terraces. With this method, it is possible to measure density changes as small as $10^{-3}$ ML for atomic species [15-17]. Intensity changes are normalized to the intensity of the clean surface at the start of the experiment. For BDA on $\mathrm{Cu}(001)$, we found in a previous study that one percent change of intensity corresponds to a density change of $5.2 \times 10^{-4} \mathrm{ML}$ [14]. The temperature of the Knudsen cell type BDA evaporator was kept constant at $446 \mathrm{~K}$ during the experiments. This setting gave a BDA deposition rate of $F=1.06 \times 10^{-6} \mathrm{ML} / \mathrm{s}$.

We first describe a typical BDA growth experiment at $332 \mathrm{~K}$. The experiment starts with a clean surface. Right after the shutter of the BDA evaporator is opened (defined as $t=0 \mathrm{~s}$ ), the BDA coverage in the dilute phase increases linearly (see Fig. 1). Molecules in the dilute phase have the lowest chemical potential up to the equilibrium density $\theta_{\text {eq }}(T)$. Once $\theta$ becomes (significantly) larger than $\theta_{\text {eq }}$, the dilute phase is supersaturated and nucleation of $c(8 \times 8)$ domains becomes possible ( $t \approx 1600 \mathrm{~s}-2200 \mathrm{~s}$ in Fig. 1$)$. However, due to the aforementioned nucleation barrier, the nucleation rate depends strongly on supersaturation. In Fig. 1, we show the measured density together with the number of islands nucleating in time windows of $100 \mathrm{~s}$. The nucleation rate depends strongly on supersaturation and is highest during the maximum $\theta(t \approx 1700-1800 \mathrm{~s})$. Finally, the island density is large enough to accommodate the arriving molecules and to reduce the supersaturation. In the end, nucleation stops and existing islands merely grow in size. The density $\theta$ at this point is almost equal to the equilibrium value $\theta_{\text {eq }}$.

We observe the decay of small nuclei throughout the distinct nucleation period [18]. The number of nuclei that decayed are shown as red (cross-hatched) bars in Fig. 1. Nuclei which contain up to 4000 molecules can decay. The time scales of these fluctuations are several seconds or even minutes. Islands that are larger are stable for a substantially longer time span. Another way for these larger islands to decay is through Ostwald ripening, which occurs on a time scale of hours or even days for the sample temperatures used here.

As can be seen in Fig. 1, about $40 \%$ of the observed nucleated clusters are unstable and eventually decay. Example LEEM images of these subcritical nuclei are shown in Fig. 2 for two experiments at 305 and $332 \mathrm{~K}$. It is difficult to capture the process in a few images. The average lifetime of the subcritical nuclei is only one or two frames, and not many of them are imaged simultaneously. We could not distinguish any specific pattern formed by the position where the unstable nuclei nucleated. Particularly, the distance to a substrate step did not seem to matter and islands did not nucleate twice at the same position. The latter would be a strong indication for defect-induced nucleation. However, we do note a tendency for extremely long-range order formed by the position where a nucleation event occurred, irrespective of whether the nucleus is stable or not. For example, in the second panel of Fig. 2(b), the central region is almost without islands, while above this region, several islands nucleated with a large density. Probably because of diffusion and/or attachment limitations [12], there is a higher nucleation probability on or close to lines connecting existing islands, leading to preferential nucleation along these line and, thus, to an average island-island distance that is smaller than what would be expected for purely random nucleation. Tentatively, we explain this observation with local density gradients in the dilute phase, which are generated in a complex way by growing and decaying islands. We already reported that 

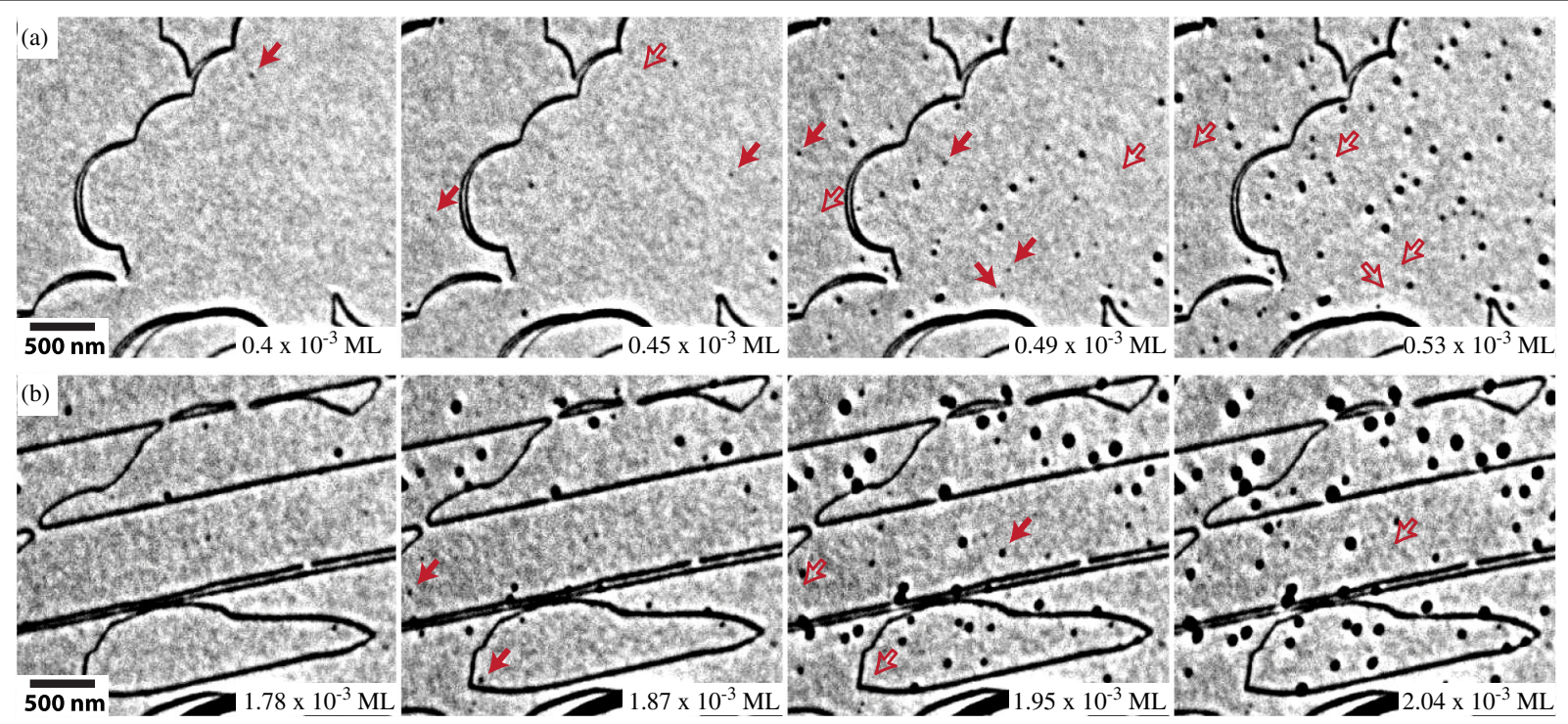

FIG. 2 (color online). LEEM images taken during the nucleation phase (total BDA coverage is indicated in the images) with $T_{S}=305 \mathrm{~K}$ (a) and $T_{S}=332 \mathrm{~K}$ (b). Islands marked with a red arrow disappeared in the subsequent image, and the corresponding position is marked with hollow arrows. Time between images is $40 \mathrm{~s}$ (a) and $80 \mathrm{~s}$ (b), respectively.

island decay is limited by diffusion, which means that local density gradients can exist $[12,14]$. The molecular density is then highest on the line connecting circular islands. Subtle differences in the local supersaturation will then dramatically influence the local nucleation rate.

We analyzed the maximum size each nucleus reached before decaying and grouped them in bins with size windows of $500 \mathrm{~nm}^{2}$. Figure 3 shows these distributions for experiments at substrate temperatures of $T=297 \mathrm{~K}$ and $T=332 \mathrm{~K}$. Obviously, the decay of larger nuclei is observed less often. For nuclei smaller than $1000 \mathrm{~nm}^{2}$, we are limited by the instrument resolution: With a field of view of $4000 \mathrm{~nm}$ and an image size of $512 \times 512$ pixels, a $1000 \mathrm{~nm}^{2}$ nucleus covers about 16 pixels. Any determination of the size of decaying islands is therefore affected by a large error bar. Especially for smaller scale fluctuations $k 1000 \mathrm{~nm}^{2}$ ), we may not capture all events. From the final nucleation density and the maximum size distributions (Fig. 3), we can calculate the probability for a nucleus of a given size to decay. These probabilities are shown in Fig. 4 for four experiments between 297 and 332 K. Nuclei that reached a size of $1500 \mathrm{~nm}^{2}$ decay with a probability between $17 \pm 3 \%(332 \mathrm{~K})$ and $8 \pm 1 \%(297 \mathrm{~K})$. As expected, the probability for a nucleus to decay is dropping for larger sizes. Except for the smallest sizes, this decay probability can be very well fitted with exponentials of the form $p_{\text {decay }}=\exp \left(-A / A^{*}\right)$. This is reasonable given that the size distribution of subcritical nuclei is also given by an exponential (Eq. (2)). From the fits, we find that nuclei with a size between $400 \mathrm{~nm}^{2}(297 \mathrm{~K})$ and $600 \mathrm{~nm}^{2}(332 \mathrm{~K})$ have an equal chance to decay or grow. This value should depend on $\Delta \mu$. We did not see such a dependence for two reasons: first, most of the islands nucleate during the largest $\Delta \mu$ anyway (see Fig. 1), and second, the statistics are too poor.
It is intriguing that we can observe critical fluctuations in such detail as we show here. Why is this possible? Compared to other systems, the fraction of molecules in the dilute phase of BDA on $\mathrm{Cu}(001)$ is already substantial at room temperature. This means that the nucleation rate will be higher at relatively small supersaturation, and the critical nucleus size accordingly very large, cf., Eq. (1). The 2D cohesive energy of the BDA molecules in the hydrogen bonded $c(8 \times 8)$ structure is rather small, which leads to high densities in the dilute phase at room temperature [14]. The combination of an appreciable nucleation rate at low supersaturation and slow molecular diffusion results in a large critical nucleus which has a sufficiently long lifetime to be observed.

Finally, we emphasize that we present a rather complete data set on the nucleation of BDA on $\mathrm{Cu}(001)$. We have the equilibrium density of the BDA molecules in the dilute phase and from the phase coexistence line of the dilute and the crystalline phase we have obtained the $2 \mathrm{D}$ cohesive energy of $0.35 \pm 0.03 \mathrm{eV}$ [14]. From that value we can
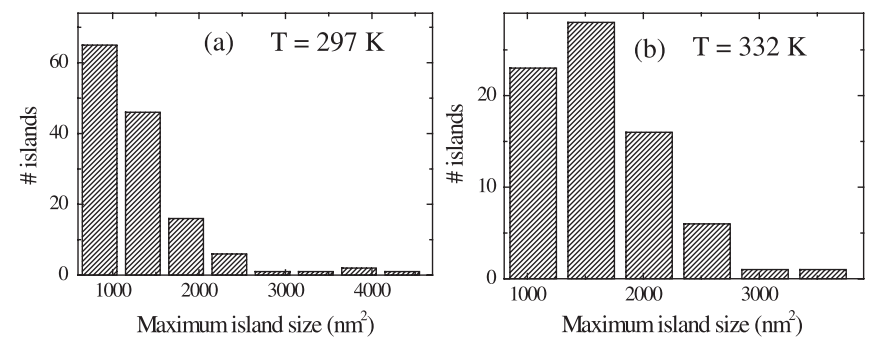

FIG. 3. Maximum island size reached by islands that decayed for two experiments at $T=297 \mathrm{~K}$ (a) and $T=332 \mathrm{~K}$ (b). The total number of stable and unstable nuclei was $381(T=297 \mathrm{~K})$ and $189(T=332 \mathrm{~K})$ in an area of $12.5 \mu \mathrm{m}^{2}$. 


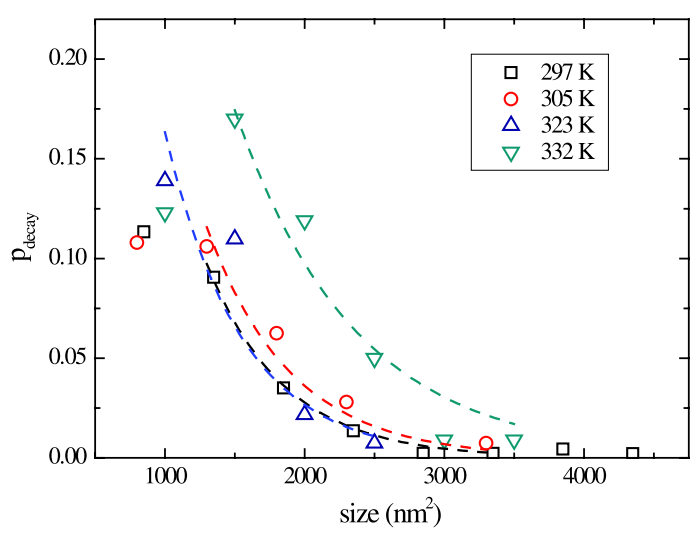

FIG. 4 (color online). Decay probability for clusters reaching a certain size for four experiments at different temperatures. The dashed lines are exponential fits to the data.

directly derive the line tension $\chi=86 \mathrm{meV} / \mathrm{nm}$. From Fig. 1 we learn that the molecular density in the dilute phase reaches $\theta_{\max }=2 \times 10^{-3} \mathrm{ML}$, while the equilibrium value is about $\theta_{\mathrm{eq}}=1.6 \times 10^{-3} \mathrm{ML}$. Treating the dilute phase as an ideal 2D monatomic gas, the maximum chemical potential difference during nucleation is then: $\Delta \mu=k T \ln \left(\frac{\theta_{\max }}{\theta_{\text {eq }}}\right) \approx 6 \mathrm{meV}$. With the molecular area $\Omega$ of $1.043 \mathrm{~nm}^{2}$, we have all the information we need to extract the Gibbs free energy for nucleation, which so far was not reported for any heteroepitaxial system. Tromp and Hannon reported a value of $1.5 \mathrm{eV}$ for high temperature homoepitaxial nucleation of $\mathrm{Si}$ on $\mathrm{Si}(001)$ [5]. Rewriting Eq. (1) for this purpose, we obtain $\Delta G^{*}=$ $\pi \frac{\Omega \chi^{2}}{\Delta \mu}$. At $332 \mathrm{~K}$, the Gibbs free energy barrier for nucleation of BDA on $\mathrm{Cu}(001)$ amounts to $4.0 \mathrm{eV}$ with an error flag of about $25 \%$, with the uncertainty dominated by those in line tension $\chi$ and $\Delta \mu$.

With the above numbers for the line tension and the chemical potential difference, we obtain a size of the critical nucleus at $332 \mathrm{~K}$ of $A^{*} \approx 680 \mathrm{~nm}^{2}$. We estimate this number to be correct within a factor of 2 due to uncertainties in $\Delta \mu$ and $\chi$, equally contributing to the possible error. This number compares favorably with the experimental value of $600 \mathrm{~nm}^{2}$ at $332 \mathrm{~K}$. This value is subject to experimental uncertainties, related to field distortions which may generate bright halos surrounding the islands. We estimated this effect by applying realistic values in Eq. (8) of reference [19] to be less than $10 \%$ for islands of $700 \mathrm{~nm}^{2}$. We therefore conclude that our data are convincingly consistent, and the critical nucleus has a large size of about 650 molecules at $332 \mathrm{~K}$. We found substantial fluctuations in the size of the unstable nuclei. Up to $4000 \mathrm{~nm}^{2}$, islands still have a finite probability to decay for a critical nucleus size of $680 \mathrm{~nm}^{2}$. These fluctuations are a lot larger than expected. In classical nucleation, it is assumed that clusters with Gibbs free energy of $\Delta G=\Delta G^{*} \pm k T$ contribute to the nucleation. In this context, the largest cluster that still decays will have a Gibbs free energy of the critical nucleus minus kT. The size fluctuations can be estimated through the so called Zeldovich factor [4], which would, however, underestimate the magnitude of the size fluctuations by one order of magnitude. We suggest that local fluctuations in supersaturation are responsible for this remarkable critical behavior. This is consistent with the diffusion limitations during decay we reported earlier [12].

In conclusion, we have measured size fluctuations of near-critical clusters during the nucleation of BDA on $\mathrm{Cu}(001)$ for temperatures between 297 and $332 \mathrm{~K}$. From the LEEM image intensity data, we extract the local chemical potential difference, which is of the order of (a few) meV. We sample the probability for decay and further growth of individual islands. By equalizing the integrated values of both probabilities, we arrive at critical island sizes of 400-600 $\mathrm{nm}^{2}$. We find that islands with a size of $1500 \mathrm{~nm}^{2}$ decay with a probability between $8 \%$ and $17 \%$, depending on the substrate temperature. The decay is exclusively observed for subcritical nuclei and in those situations where the surrounding dilute phase is still supersaturated. It is remarkable that islands with a size of 6 and 4 times the critical value have still a 1\%-2\% and $>10 \%$ probability, respectively, of eventual decay. We believe that this commonly neglected behavior is generic, especially in the case of low supersaturation. Finally, the complete set of microscopic data allowed us to determine experimentally the Gibbs free energy for nucleation. For BDA growth on $\mathrm{Cu}(001)$ at $332 \mathrm{~K}$ and a chemical potential difference of $6 \mathrm{meV}$, we obtain a value of $\Delta G=4.0 \mathrm{eV}$ with an uncertainty of about $25 \%$. This method using LEEM can be applied in general.

[1] M. Zinke-Allmang, L. C. Feldman, and M.H. Grabow, Surf. Sci. Rep. 16, 377 (1992).

[2] H. Ibach, Physics of Surfaces and Interfaces (Springer, New York, 2006), 1st ed., p. 658.

[3] J.A. Venables, Introduction to Surface and Thin Film Processes (Cambridge University Press, Cambridge, 2000).

[4] I. V. Markov, Crystal Growth for Beginners (World Scientific, Singapore, 2003), 2nd ed., Chap. 2.

[5] R. M. Tromp and J. B. Hannon, Surf. Rev. Lett. 9, 1565 (2002).

[6] W. Theis and R. M. Tromp, Phys. Rev. Lett. 76, 2770 (1996).

[7] I. Goldfarb, Phys. Rev. Lett. 95, 025501 (2005).

[8] D. E. Jesson, M. Kästner, and B. Voigtländer, Phys. Rev. Lett. 84, 330 (2000).

[9] B.-S. Lee, G. W. Burr, R. M. Shelby, S. Raoux, C. T. Rettner, S. N. Bogle, K. Darmawikarta, S. G. Bishop, and J. R. Abelson, Science 326, 980 (2009).

[10] U. Gasser, E. R. Weeks, A. Schofield, P. N. Pusey, and D. A. Weitz, Science 292, 258 (2001).

[11] J. A. Venables, G. D. T. Spiller, and M. Hanbucken, Rep. Prog. Phys. 47, 399 (1984).

[12] F. S. Khokhar, R. van Gastel, D. Schwarz, H. J. W. Zandvliet, and B. Poelsema, J. Chem. Phys. 135, 124706 (2011). 
[13] S. Stepanow, N. Lin, F. Vidal, A. Landa, M. Ruben, J. V. Barth, and K. Kern, Nano Lett. 5, 901 (2005).

[14] D. Schwarz, R. van Gastel, H. J. W. Zandvliet, and B. Poelsema, Phys. Rev. B 85, 235419 (2012).

[15] E. Loginova, N.C. Bartelt, P. J. Feibelman, and K. F. McCarty, New J. Phys. 11, 063046 (2009).

[16] E. Loginova, N. C. Bartelt, P. J. Feibelman, and K. F. McCarty, New J. Phys. 10, 093026 (2008).
[17] J. de la Figuera, N. C. Bartelt, and K. F. McCarty, Surf. Sci. 600, 4062 (2006).

[18] See Supplemental Material at http://link.aps.org/ supplemental/10.1103/PhysRevLett.109.016101 for a short movie visualizing the critical fluctuations during the nucleation at $305 \mathrm{~K}$.

[19] S.A. Nepijko, N.N. Sedov, and G. Schönhense, J. Microsc. 203, 269 (2001). 